\title{
Alentando la autonomía en la educación superior: modelo de un centro de autoaprendizaje de lenguas ${ }^{1}$
}

Maria Giovanna Tassinari

Freie Universität Berlin

Giovanna.Tassinari@fu-berlin.de

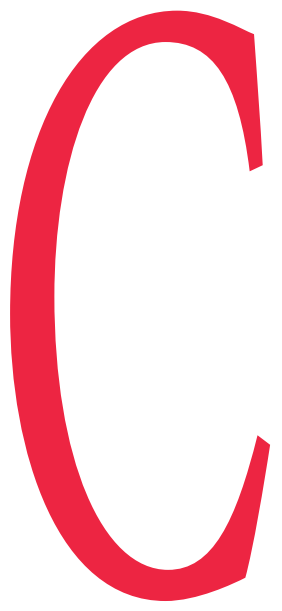

uando comencé a trabajar en la autonomía en 2004, al fundar el Centro de autoaprendizaje de lenguas (CILL) en la Freie Universität de Berlín (FUB), pensé, bastante ingenuamente, que dar oportunidades a los estudiantes para dirigir su propio aprendizaje sería suficiente para fomentar su autonomía. Curiosamente, parecía haber olvidado que, además de dar oportunidades, otros factores pueden fomentar u obstaculizar el desarrollo de la autonomía, entre otros, factores internos como las creencias y las actitudes hacia el aprendizaje, factores afectivos e individuales, y factores externos como el entorno del aprendizaje, el contexto institucional y social. En otras palabras, visto desde la perspectiva de los sistemas adaptativos complejos (CAS), la autonomía necesita una comunidad, una comunidad de aprendizaje y de práctica, para ser ejercida por los individuos en un contexto dado.

Tardé en darme cuenta de que la autonomía solo puede florecer en un entorno en el que los actores del contexto educativo cooperen hacia objetivos educativos comunes. Además me percaté de que para fomentar la autonomía era necesario esforzarse significativamente para alcanzar una concepción común negociada con el personal académico y los estudiantes.

En este artículo describiré cómo abordo este reto con la ayuda de estructuras y recursos establecidos en el CILL. Empezaré por definir brevemente la autonomía desde una perspectiva de CAS (Sección 1), posteriormente explicaré por qué elijo el verbo "alentar" la autonomía (Sección 2). A continuación describiré el contexto del CILL, su función dentro de la institución (Sección 3). Después, ilustraré el trabajo con el personal del CILL, los tutores y los docentes, y su repercusión en el desarrollo de la autonomía (Sección 4), así como las ventajas de constituir una comunidad de práctica más amplia (Sección 5). Algunas reflexiones sobre los requisitos para una comunidad de práctica de éxito (Sección 6) y finalmente algunas recomendaciones (Sección 7) concluirán mi contribución.

\section{Definición de autonomía}

La autonomía ha sido definida y descrita de manera diferente por varios investigadores (entre otros, Benson, 2011; Holec, 1981, 2009; Little, 1991, 2015; Littlewood, 1996, 1999; Oxford, 2003; Martinez, 2008). Centrándose en el aprendizaje de idiomas, la autonomía se asocia a la noción de acción independiente en el proceso de aprendizaje. La acción independiente es eficaz si los 


\section{Resumen:}

Implementar la autonomía en el aprendizaje de idiomas y en la enseñanza en la mayoría de los contextos institucionales, después de décadas de investigación y práctica, sigue siendo un desafío para docentes y estudiantes. Enfrentarse a estos desafíos puede resultar más fácil, si estos no se hacen de forma aislada sino más bien como parte de una comunidad de aprendizaje y enseñanza. Mi experiencia en el Centro de autoaprendizaje de lenguas (Centre of Independent Language Learning, CILL) en el Centro de Idiomas de la Freie Universität Berlin (FUB) es que el primer paso para implementar la autonomía es alentarla construyendo una comunidad de práctica. Para los docentes, una comunidad de práctica significa la oportunidad de debatir sobre su concepción de la autonomía, compartir y reflexionar sobre experiencias con enfoques o tareas, con el fin de desarrollar estrategias comunes para la enseñanza, y si fuera necesario, para abordar aspectos de gestión / administración.

Una comunidad de práctica en la que los docentes y los estudiantes intercambien sus puntos de vista y cooperan hacia una concepción común de enseñanza y aprendizaje autónomos ofrece una mejor oportunidad para construir una cultura de aprendizaje basada en la autonomía. En el CILL se crean oportunidades para tal intercambio, involucrando a estudiantes y docentes con el objetivo tanto de reflexionar sobre la oferta del CILL como de ayudar a desarrollarla aún más.

Palabras clave: autonomía, aprendizaje de idiomas, centros de idiomas de libre acceso, comunidad de práctica, educación superior

estudiantes son capaces de tomar decisiones informadas y reflexivas sobre su aprendizaje y pueden hacerlo en el contexto institucional y social en el que viven y aprenden. La capacidad, la libertad y la acción están, por lo tanto, en el núcleo de la autonomía. La reflexión y la metacognición, así como la capacidad de interactuar y negociar con otros durante el proceso de aprendizaje, son esenciales para ejercer la autonomía hasta cierto punto (Little, 1991, 2001a 2001b).

Con un enfoque específico en el alumno, esta definición puede ser operativizada identificando las diferentes dimensiones de este complejo constructo con el fin de ayudar a los estudiantes y docentes en el proceso de desarrollo y ejercicio de esta capacidad. Estas dimensiones son:

- cognitiva y metacognitiva (conocimiento cognitivo

\section{Abstract:}

Implementing autonomy in language learning and teaching in most institutional contexts is still, even after decades of research and practice, a challenge for teachers and learners. Facing these challenges may be easier, if this is done not in isolation, but rather as part of a learning and teaching community.

My experience at the Centre for Independent Language Learning (CILL) at the Language Centre of the Freie Universität Berlin is that the first step towards implementing autonomy is encouraging it while building a community of practice. For teachers, a community of practice means the opportunity to discuss their understanding of autonomy, to share and reflect experiences, to develop common strategies both for teaching and for addressing the administration, if need be.

An even better opportunity to build a learning culture based on autonomy is to support teachers and learners to exchange their perspectives and cooperate towards a common understanding of (more) autonomous teaching and learning. One of the aims of the CILL is to create opportunities for such an exchange, involving student assistants and teachers to reflect on the provision of the CILL and to help to further develop it.

Keywords: autonomy, language learning, self-access language centres, community of practice, higher education y metacognitivo sobre el lenguaje, sobre el aprendizaje del lenguaje, sobre uno mismo, sus creencias, concienciación, reflexión);

- motivacional (voluntad y motivación para actuar de forma autónoma);

- orientada a la acción (decisiones relacionadas con el proceso de aprendizaje, medidas tomadas, estrategias, habilidades, conductas de aprendizaje);

- afectiva (los sentimientos y las emociones de los estudiantes que se relacionan con el proceso de aprendizaje del idioma y la forma de tratarlos);

- social (capacidad de negociación en la interacción no sólo con asesores, profesores y hablantes nativos, sino también entre iguales).

Estas dimensiones se ilustran en el modelo dinámico 
de autonomía, una herramienta para la reflexión y la autoevaluación que desarrollé como un proyecto de doctorado (Tassinari, 2010, http://www.sprachenzentrum. fu-berlin.de/slz/index.html). En el modelo se muestran como "esferas" que representan competencias, habilidades, actitudes, creencias, procesos de toma de decisiones y comportamientos de aprendizaje. Están estrechamente relacionadas entre sí y se desarrollan dentro de una dinámica compleja.

La autonomía como capacidad es un sistema adaptativo complejo (CAS), un sistema autoorganizado, compuesto por múltiples elementos interrelacionados, cada uno de los cuales influye y es influido por los demás; como parte del CAS, el contexto está igualmente involucrado en estas múltiples interacciones. Más que la suma de sus partes, un CAS funciona como un todo, cuyos elementos están involucrados en dinámicas permanentes (Larsen-Freeman y Cameron, 2003, citado en Mercer, 2014: 163). Los CAS son "dynamic, chaotic, unpredictable, non-linear, adaptive, open, self-organizing, and sensitive to initial conditions and feedback" (Paiva, 2006, citado en Paiva y Braga, 2008: 447). De la misma manera, el desarrollo de la autonomía es dinámico y no lineal, y está en relación con múltiples factores.

En contextos institucionales, los elementos que concurren en la autonomía son los actores de los entornos educativos: estudiantes, profesores, asesores o iguales, con sus características individuales, experiencias, creencias, emociones, comprensión de sus papeles y sus comportamientos; además, las estructuras institucionales, los cursos y los currículos; los materiales de aprendizaje y los recursos. Por lo tanto, "complex-systems interpretation of autonomy does not situate the development of autonomy solely within individuals, but it recognises its emergence from the interaction of different individuals together and in relationship with others and their affordances" (Tatzl, 2016: 47)

Esto explica por qué, como destacaron anteriormente muchos investigadores, la autonomía fluctúa constantemente dentro de un continuo de autonomía y heteronomía (Everhard, 2015). Las vías hacia la autonomía son diferentes para cada individuo y a veces impredecibles (Stewart y Irie, 2012).

La autonomía no se circunscribe solo a los estudiantes, sino queincluyea los docentes. Enuna concepción integrada de la autonomía del estudiante y del docente, se define como "the competence to develop as a self-determined, socially responsible and critically aware participant in (and beyond) educational environments, within a vision of education as (inter)personal empowerment and social transformation" (Jiménez Raya, Lamb y Vieira, 2017: 1). De manera similar a los estudiantes, los docentes que se comprometen a desarrollar su propia autonomía y fomentar la autonomía del alumno necesitan el apoyo de una comunidad para compartir la comprensión y la reflexión sobre su práctica profesional. En esta concepción se sustenta el marco pedagógico y mi trabajo diario en el CILL.

\section{Alentar la autonomía: ¿sigue siendo un reto?}

Para describir el trabajo con los tutores, docentes y estudiantes en el CILL, prefiero usar la palabra "alentar" en lugar de "fomentar" la autonomía, ya que los primeros pasos hacia la autonomía requieren que los individuos sientan que se les infunde ánimo y energía para cambiar, desarrollarse hacia diferentes formas de aprender y / o enseñar, y sentirse libre de hacerlo. Mis acciones como directora del centro de autoaprendizaje de lenguas apuntan más bien a alentar, no a imponer comportamientos (más) autónomos a los estudiantes, tutores, docentes o a mi misma. Una atmósfera de libertad, responsabilidad y apoyo es uno de los factores determinantes para estimular la motivación de los estudiantes y los docentes para participar en este desafiante proceso. Establecer y mantener una atmósfera de este tipo en un contexto institucional es el primer reto, dados los requisitos institucionales y la presión bajo la que se enseña y aprende.

Alentar la autonomía requiere la voluntad y la capacidad de motivar a los estudiantes, para ayudarles a reorientarse, si así lo desean, teniendo en cuenta que esta reorientación puede ser motivadora, pero también exigente, desestabilizadora, ya que puede verse facilitada u obstaculizada por varios elementos del sistema. Alentar la autonomía significa también involucrar e inspirar a los docentes, hacer preguntas incómodas, iniciar en nosotros mismos y en otros actores del proceso educativo la reflexión, la conciencia crítica, la autoevaluación y el deseo de indagar. Por último, pero no por ello menos importante, alentar la autonomía requiere una reflexión personal, consciente de que la autonomía no es "un estado de equilibrio" al que hay que llegar una y otra vez (Little, 1991), sino que, por el contrario, las recaídas son posibles y deben ser tratadas.

En las siguientes secciones ilustraré brevemente cómo se fomenta este proceso de cambio para el personal que trabaja en el centro de autoaprendizaje de lenguas de CILL (tutores), los estudiantes y los docentes. 


\section{El Centro de Autoaprendizaie de Lenguas en la FUB}

El CILL es parte del Centro de Lenguas de la FUB. El Centro de Lenguas proporciona módulos de idiomas tanto para especialistas como para no especialistas en 12 idiomas. Los objetivos de aprendizaje, los contenidos y las formas de enseñanza y aprendizaje, así como los exámenes finales se definen en la normativa de estudios. Además de las horas presenciales (en general 4 horas por semana, aproximadamente $1 / 3$ de la carga de trabajo), los módulos de idioma requieren aprendizaje no presencial (alrededor de $2 / 3$ de la carga de trabajo) en forma de aprendizaje independiente y cooperativo, en estrecha retroalimentación con los profesores (normativa de la FUB 11/2015: http://www.fu-berlin.de/service/zuvdocs/ amtsblatt/2015/ab112015.pdf?1431335088).

Por esta razón el fomento de la autonomía, el aprendizaje independiente y cooperativo debería ser parte de cada módulo de lenguaje.

El CILL se creó en 2005 para ayudar a los estudiantes matriculados en un módulo de lengua a organizar la carga de trabajo en las horas no presenciales y a los estudiantes que aprenden un idioma en un modo de aprendizaje autodirigido. Sin embargo, la misión del CILL es fomentar tanto la autonomía del alumno como la del profesor y el aprendizaje del idioma. Esto se establece claramente en el sitio web CILL, con una breve definición de mi concepción de la autonomía (http://www.sprachenzentrum.fu-berlin. de/slz/das-slz/lernerautonomie/index.html).

Por autonomía entendemos la capacidad y la oportunidad de:

- Tomar decisiones sobre el aprendizaje de manera independiente (¿Qué quiero aprender? ¿Cómo quiero aprender?) y de implementar estas decisiones

- Poner su propia motivación en el centro de su aprendizaje, seguir intereses personales, preferencias y su propia creatividad

- Aprender a través de interacciones sociales

- Reflexionar sobre el propio aprendizaje

- Tomar conciencia de los límites y de las posibilidades con los que se aprende y se vive

- Pensar de manera crítica

- Desarrollar aún más la propia identidad como persona que tiene voz en las decisiones y actúa socialmente.

Por autonomía del docente entendemos:

"the competence to develop as a self-determined, socially responsible and critically aware participant in (and beyond) educational environments, within a vision of education as (inter)personal empowerment and social transformation"

(Jiménez Raya, Lamb y Vieira 2017: 1).

El CILL ofrece recursos y asistencia para aprender más de 20 idiomas extranjeros y se basa en los siguientes pilares:

1. Una variedad de materiales y recursos impresos, DVD, recursos de audio y software;

2. Un apoyo para el aprendizaje autónomo de idiomas en forma de guías de estudio, talleres y un servicio de asesoramiento lingüístico;

3. Un sitio web con un catálogo específico para el aprendizaje de idiomas, directrices para el aprendizaje autónomo, una amplia colección de enlaces para el aprendizaje de idiomas (véase http://www. sprachenzentrum.fu-berlin.de/slz/index.html);

4. Un programa de intercambio lingüístico (tándem);

5. Apoyo para el aprendizaje social en forma de tutoriales, noches de juegos y espacio para grupos de aprendizaje autoorganizados.

En el CILL, los estudiantes pueden trabajar con materiales impresos, materiales auténticos, ver películas en DVD, escuchar audiolibros, leer revistas, aprender gramática - en el caso de Alemán como lengua extranjera hay a disposición una amplia colección de hojas con ejercicios gramaticales para llevar-, consultar diccionarios o libros de referencia, utilizar ordenadores y puestos de trabajo de vídeo. También se proporcionan espacios para aprender en grupo y para conversar. Aunque los módulos de idioma incluyen un elemento de aprendizaje independiente y cooperativo, la asistencia al CILL no es obligatoria y puede negociarse con el docente. Algunos estudiantes usan el CILL para un trabajo de recuperación, otros crean un plan de aprendizaje individual; algunos aprenden en tándem, otros trabajan en un proyecto, otros acuden a una sesión de asesoramiento lingüístico, ya sea por sugerencia de su docente, ya sea por iniciativa propia.

El servicio de asesoramiento de idiomas es opcional y está abierto a estudiantes de todos los idiomas. Los estudiantes pueden reservar una o más sesiones de asesoramiento en las que se les anima a reflexionar sobre su proceso de aprendizaje y se les apoya en la toma de decisiones, en la elección de materiales y tareas apropiadas y en la evaluación de su progreso y / o su proceso de aprendizaje. El personal de CILL está compuesto por la directora, una bibliotecaria y trece tutores.

\subsection{El CILL para profesores}

Aunque en sus inicios el CILL fue pensado como apoyo 
únicamente para estudiantes, desde mi propia experiencia me di cuenta de que los docentes también requerían apoyo en la búsqueda de enfoques para organizar la carga de trabajo no presencial de los estudiantes y, de manera más general, para desarrollar la autonomía de los estudiantes. El apoyo a los docentes es por lo tanto una peculiaridad del CILL y consiste en ofrecer a los profesores del Centro de Idiomas y de los Departamentos de Filología talleres sobre temas relacionados con la autonomía del profesor y del estudiante, asesoramiento individual sobre proyectos, si fuera necesario, y espacios y oportunidades para reflexionar sobre la práctica de la enseñanza de idiomas, compartir experiencias, experimentar y reflexionar sobre cómo promover autonomía en el aprendizaje de idiomas.

Un pequeño grupo de trabajo compuesto por algunos docentes y yo misma como directora del centro de autoaprendizaje de lenguas trabaja en el desarrollo de acciones para vincular mejor la enseñanza y el aprendizaje en el aula y el aprendizaje fuera del aula.

\section{ClLL: hacia una comunidad de práctica}

En el marco de una teoría social del aprendizaje (Wenger, 1998), una comunidad de práctica está constituida por un grupo de actores pertenecientes a una misma organización que trabajan y se apoyan mutuamente dentro de un marco (de trabajo) determinado. Para una comunidad de práctica es esencial el desarrollo del aprendizaje individual y social, compartiendo conocimientos, experiencias, recursos y perspectivas significativos (Wenger, 1998: 5). A diferencia de otros grupos de trabajo, las comunidades de práctica en general se autoorganizan y no son controladas directamente por la administración de una organización.

Según Wenger, los elementos necesarios para el éxito de una comunidad de práctica son: un área de experiencia compartida (el dominio), un grupo de actores que interactúa regularmente (la comunidad) y recursos que se pueden compartir (la práctica) (Wenger, 2006, citado en Gardner y Miller, 2014: 107).

El CILL involucra en su trabajo diario y en su desarrollo a varios actores del contexto educativo: la directora, la bibliotecaria, los tutores del centro, y, en menor medida, los profesores y el resto de estudiantes. Todos estos actores aportan su propia perspectiva y experiencia al proyecto y contribuyen así al entorno de aprendizaje. Por lo tanto, como ilustran Gardner y Miller (1999: 11), el centro de autoaprendizaje de lenguas está constituido y se desarrolla a través de la interacción de los diversos participantes.

Dado que el CILL está bajo mi dirección y forma parte de una organización superior, no puede definirse per se como una comunidad de práctica. Sin embargo, tiene la oportunidad de convertirse en una, si la interacción entre los participantes se basa en intereses y objetivos comunes, y si yo, como directora, garantizo espacios de decisión y acción autónoma.

A continuación, describiré brevemente cómo el trabajo con los tutores, profesores y estudiantes alienta la autonomía de estos actores y contribuye a seguir desarrollando la provisión del CILL. Posteriormente voy a reflexionar sobre cómo se podría fomentar el desarrollo de una comunidad de práctica en el CILL.

\subsection{Crecer con los tutores}

Los trece tutores en el CILL tienen como tareas: dar la bienvenida a los estudiantes, ofrecerles la primera información sobre el CILL, mantener y desarrollar el sitio web, llevar a cabo el programa en tándem (asociaciones de idiomas), entre otras tareas. Son contratados para un puesto de dos años de duración, que en algunos casos puede ser renovado por otros dos años, de modo que algunos de ellos llegan a permanecer hasta cuatro años. Trabajan aproximadamente 40 horas al mes, 10 horas por semana. Son seleccionados preferentemente entre estudiantes de Filología o de ciencias de la Educación, y, en general, hablan más de un idioma; algunos de ellos son alemanes, otros vienen a vivir y estudiar a Berlín desde diferentes países. El equipo de tutores es por lo tanto muy diverso; cada uno de ellos tiene competencias, intereses y habilidades particulares que pueden ser muy útiles para desarrollar aún más la provisión del CILL. Debido a su doble estatus de estudiantes y de personal del CILL, los tutores aportan perspectivas estimulantes al trabajo del CILL.

Ante de empezar en el CILL, reciben una formación inicial. Esta incluye una introducción al CILL, su marco pedagógico, los recursos y las tareas de los tutores. Una vez incorporados se les ofrecen talleres pedagógicos, de manera regular, sobre temas como mejorar la comunicación con los usuarios del CILL, analizar materiales de aprendizaje y describirlos en el catálogo en línea, analizar recursos en línea para el aprendizaje de idiomas, contribuir a una wiki o el asesoramiento entre iguales para el aprendizaje de idiomas. Los temas se negocian con los tutores de acuerdo a sus necesidades y / o prioridades en el desarrollo del CILL. Algunos de los talleres están dirigidos por ellos mismos. Además, se forman equipos más reducidos formados por los encargados de ámbitos específicos del trabajo en el CILL como el sitio web, el programa en tándem o el asesoramiento entre iguales. 
Comodirectora delCILL, mitarea consiste en identificar, junto con cada uno de los tutores, sus competencias, su área de interés y sus prioridades para su desarrollo personal y profesional. A partir de aquí, decidimos de manera conjunta qué tareas o proyectos estarán a cargo de quién. Es esencial para mí alentar su autonomía, dándoles espacio para la elección y la experimentación; a continuación les ofrezco mi apoyo y orientación para implementar el proyecto; finalmente, debatimos y evaluamos juntos los resultados y el proceso en sí. Hasta la fecha, ya se han implementado varios proyectos en CILL a iniciativa de tutores, como el rediseño del encuentro en tándem, la planificación de una noche de juegos para parejas tándem, el asesoramiento entre iguales para el alemán como lengua extranjera, el desarrollo de un nuevo concepto para la formación inicial de los nuevos tutores, o el desarrollo de nuevas guías de estudio.

Incluso si algunos de los proyectos no se pueden llevar a cabo de manera regular (debido a restricciones externas como puede ser la finalización del contrato del tutor a cargo), todos ellos contribuyen a la ampliación de la oferta educativa del CILL y hacen que los tutores se sientan responsables de su trabajo como agentes decisivos en el CILL. Es gratificante ver cómo los tutores colaboran entre sí, inician proyectos juntos, comparten sus competencias y experiencias y se ayudan unos a otros dentro y fuera de su trabajo en el CILL.

El trabajo realizado durante estos años me ha hecho ver que trabajar de esta manera con los tutores nos permite aprender unos de otros. Personalmente, he aprendido a respetarles y a confiar en ellos, a escuchar atentamente lo que dicen y a reconocer su contribución única al CILL. También me he dado cuenta de que, preocupada por garantizar la rutina diaria en el CILL y teniendo que empezar de nuevo la formación básica y la supervisión de los nuevos tutores de forma regular, cuando estos comienzan su prestación, a veces tiendo a controlar demasiado su trabajo y, por lo tanto, a limitar su espacio de maniobra. Afortunadamente, el diálogo abierto con los tutores les permite abordar este tema, de modo que, conscientes de ello, yo/nosotros podemos cambiar el curso de acción. Para mí personalmente, uno de los desafíos, a este respecto, es reconocer las situaciones en las que ejerzo demasiado control y encontrar la forma de ceder a los tutores más espacio de maniobra.

\section{2 Aprender de los estudiantes}

En el CILL también veo a muchos estudiantes diariamente. Con algunos de ellos solo intercambio algunas palabras cuando llegan al CILL o se van después de una sesión de estudio. Con otros, los que acuden al servicio de asesoramiento lingüístico, me involucro en un diálogo real. Estos estudiantes también contribuyen a veces a la comunidad de aprendizaje del CILL. Escuchar sus historias, las estrategias que describen, su experiencia única amplía mi perspectiva profesional y personal sobre la autonomía de aprendizaje de idiomas y fluye imperceptible en mi propia competencia como asesora. Además, como algunas de estas experiencias son compartidas y reflexionadas con docentes, contribuyen a desarrollar la competencia colectiva de la comunidad de aprendizaje del CILL.

Esto no solo me sucede a mí, sino también a los tutores: a menudo presencio conversaciones entre ellos y algunos compañeros, compartiendo experiencias de aprendizaje, evaluando recursos, o simplemente interesándose el uno por el otro. Estos intercambios espontáneos hacen del CILL un lugar donde los estudiantes pueden encontrar y construir una comunidad.

\subsection{Animar a los profesores a una mayor autonomía}

Trabajar con los docentes es crucial para integrar un centro de autoaprendizaje de lenguas en el contexto educativo institucional. De mi nombramiento como directora del CILL recuerdo a una colega, directora de un Centro de Idiomas con un innovador centro de autoaprendizaje de lenguas, que me recomendó "involucrar a los profesores".El Centro de Idiomas de la FUB emplea a cerca de 100 profesores de idiomas, de los cuales alrededor del $40 \%$ están a tiempo completo o parcial con contrato permanente y el $60 \%$ trabaja free lance. Por lo tanto, como directora del centro de autoaprendizaje de lenguas, no solo involucrar a los profesores de idiomas en el CILL, sino también animarlos a participar en el intercambio de experiencias sobre la autonomía es una empresa.

Al principio, cuando se creó el CILL, un grupo de profesores de diferentes idiomas participó en el debate para validar el marco pedagógico del CILL, los criterios para los recursos y el catálogo (véase Tassinari, 2016).

Posteriormente, cuando el CILL se puso en marcha, se invitó a los docentes a participar en diferentes actividades del CILL tales como describir materiales para el catálogo en línea, sugerir sitios web para nuestra colección de enlaces de idiomas, ofrecer asesoramiento de idiomas o talleres para tándem de idiomas. Los docentes involucrados en estas actividades obtuvieron una reducción de su carga docente $y$, en el caso de docentes free lance, un contrato extra por el trabajo realizado. Después de varias formaciones sobre la autonomía del estudiante, el aprendizaje autodirigido y el asesoramiento de idiomas, durante los años 2006-2008, un 
equipo de cinco docentes se hizo cargo, además de impartir sus cursos, del asesoramiento sobre la lengua en el CILL.

Desafortunadamente, debido a diferentes circunstancias, este equipo ya no está disponible: algunos abandonaron la universidad y otros fueron asignados a otros departamentos. También se produjeron cambios en los criterios de reducción de carga docente, asignada principalmente para tareas administrativas, de modo que actualmente los docentes que cooperan con el CILL lo hacen a título personal y sin ninguna compensación. Estos factores han reducido innegablemente el número de profesores activamente involucrados en el desarrollo de la oferta de CILL.

Negociar con la dirección del Centro de Idiomas y de la universidad para modificar esta situación es uno de los retos actuales para asegurar el desarrollo de la oferta formativa del CILL

El trabajo con los docentes consiste en ofrecer regularmente talleres sobre diversos aspectos de la autonomía de docentes y estudiantes, tanto como un tipo de formación, como una oportunidad para compartir experiencias; además, estoy disponible para consultas individuales y apoyo para proyectos relacionados con la autonomía. Un grupo de trabajo de docentes se constituyó en 2014 con el fin de desarrollar aún más el vínculo entre el aprendizaje en el aula y fuera del aula.

Trabajar con los docentes es esencial y beneficioso para mí, ya que me hizo darme cuenta de que, en lugar de proponer mi concepción de la autonomía del estudiante, es crucial que compartamos y reflexionemos sobre las diferentes formas de entender la autonomía. Este trabajo podría ayudar, como señalan Stewart y Irie, a lograr la autonomía, porque es necesario "look beyond the surface to inquire how and what forms localized pedagogy for autonomy may take" (Stewart y Irie, 2012: 1). Además, negociar un entendimiento común sobre qué es la autonomía y cómo el Centro de Idiomas de nuestra universidad puede contribuir pedagógicamente ayuda a desarrollar un enfoque pedagógico compartido, $\mathrm{y}$ es constructivo tanto para los docentes como para los estudiantes.

Del trabajo con los docentes en los últimos diez años, destacan las siguientes áreas cruciales para alentar su desarrollo hacia una mayor autonomía:

- Reflexionar sobre y / o reorientar las propias creencias $y$ actitudes

- Reflexionar sobre la concepción de la autonomía de cada uno

- Reflexionar sobre la propia disposición y motivación hacia el cambio y la autonomía

- Buscar oportunidades para el desarrollo profesional

- Desarrollar aún más la competencia propia para utilizar críticamente las oportunidades de aprendizaje en línea fuera del aula y en línea

- Tratar con las limitaciones sociales e institucionales

- Encontrar espacios de posible acción (el espacio de posibilidad superando obstáculos externos y resistencias internas, Jiménez Raya, 2017).

(para este listado véase también Tassinari, 2017a)

Desde que en los últimos años se ha producido un cambio en el profesorado del Centro de Idiomas, con la salida de algunos profesores y la entrada de nuevos, el intercambio y la reflexión se ha vuelto más difícil: por lo tanto, reunir a profesores con diferente procedencia cultural, experiencia docente y necesidades de desarrollo profesional requiere un esfuerzo aún mayor. A pesar de estos factores, una reflexión común es esencial tanto para integrar las instalaciones del CILL en la práctica de aprendizaje y enseñanza como para fomentar una pedagogía de la autonomía.

\section{Aprovechando la fuente: una comunidad extendida}

Gardner y Miller (2014) ilustran cómo se puede construir una comunidad de práctica más allá de las paredes de un centro de autoaprendizaje de lenguas singular. En Hong Kong, donde a principios de la década de 1990 se establecieron varios centros de autoaprendizaje de lenguas, los intercambios regulares entre los profesores implicados en el aprendizaje en este centros dieron lugar primero a una red y posteriormente a una organización llamada Hong Kong Association for Self-Access Learning and Development (HASALD).

"The establishment of the CoP [community of practice] along with the continued development of SALL [self-access language learning, MGT] have transformed Hong Kong into a centre of excellence in research and publication about SALL, SACs, [self-access centres, MGT] and learner autonomy". (Gardner y Miller, 2014: 106).

A la vista de mi experiencia, el intercambio con colegas, profesores de idiomas, asesores y profesionales en centros de autoaprendizaje de otras instituciones es crucial para reflexionar sobre mi propia concepción de la autonomía y mi propia práctica. Me beneficio de las discusiones con colegas de otras instituciones en diversas circunstancias, en 
conferencias, talleres y formación de profesores. Además, participar en talleres, formaciones, conferencias, asistir a eventos de grupos de especial interés sobre autonomía, como el Learner Autonomy Special Interest Group de IATEFL (https://lasig.iatefl.org/), la Independent Learning Association (https://ila2018.org/), el AILA Research Network on Learner Autonomy (https://ailarenla.org/), me proporciona inspiración e impulsos para desarrollar una postura crítica y renovar mi propia práctica.

Esta comunidad está constituida por colegas e investigadores que he conocido en conferencias, cuyos documentos o libros leo y con los que comencé a hablar, desde Nueva Zelanda a Japón, México, o Canadá. Comunicarse con ellos, reflexionar con ellos, compartir ideas y enfoques, ayuda a fortalecerme y ampliar mi perspectiva, encontrar nuevas formas de abordar cuestiones a las que pueda enfrentarme y seguir mejorando mi trabajo. Ser parte de esta comunidad es, para mí, irremplazable.

Recientemente, algunas redes se están constituyendo en el contexto alemán y europeo: la red AKS (Arbeitskreis der Sprachenzentren, http://www.aks-sprachen.de/) de profesionales de centros de autoaprendizaje tiene el propósito de compartir recursos, reflexionar sobre prácticas, discutir los criterios de calidad para centros de autoaprendizaje y el aprendizaje fuera del aula, y explorar vías para su desarrollo futuro. Además, se ha establecido un grupo sobre la autonomía dentro de CercleS con el objetivo de hacer un mapa del estado de la cuestión y de promover la autonomía en los Centros de Idiomas en la Educación Superior (www.cercles.org). Las conferencias periódicas sobre el asesoramiento lingüístico (Dresde, 2012, Hannover, 2015, Potsdam, 2017) ofrecen la oportunidad de reflexionar sobre las mejores prácticas e investigar aspectos de este nuevo papel en el panorama de la enseñanza de idiomas (véase Berndt y Deutschmann, 2014; Böcker, Saunders, Koch y Langner, 2017).

\section{Cumplir con los requisitos para una comunidad de práctica}

Reflexionando sobre mi experiencia, y la manera como trabajo en el CILL, me pregunto si las oportunidades de intercambio que ofrezco a los profesores y los tutores pueden considerarse encaminadas hacia una comunidad de práctica en la que cada uno de los participantes tiene su propia voz o si, por el contrario, sólo estoy tratando de convencer a mis colegas de que acepten la idea de la autonomía. En cualquier caso, una comunidad de práctica es más que un grupo de personas que comparten ideas y recursos en un dominio determinado. Como Gardner y Miller (2014: 108) señalan:
“[... ] a CoP [community of practice, MGT] is not a loose association of like-minded individuals sharing some ideas. In its most successful form it is a planned community which is designed for evolution with varying levels of participation where less experienced members may apprentice themselves to more experienced members who mentor them. A successful CoP will provide opportunities for interaction among members in small and large groups and also interaction with the outside world as a way of retaining perspective and learning new things. Most importantly, a successful $\mathrm{CoP}$ will evolve a rhythm for its activities and develop a balance of familiar and novel events which makes its membership comfortable but not complacent"

Para asegurar su desarrollo, una comunidad de práctica necesita cumplir con los siguientes requisitos (Gardner y Miller, 2014:108):

1. Un diseño para la evolución (lo suficientemente flexible como para permitir cambios en su desarrollo);

2. Un diálogo entre la perspectiva interna y externa (entre los miembros de la comunidad y de fuera);

3. Diferentes niveles de participación (para miembros que son más o menos activos dentro de la comunidad);

4. Espacios comunitarios públicos y privados (para "una variedad de oportunidades de interacción" para fortalecer la comunicación dentro de la comunidad);

5. Centrarse en el valor (discusión explícita sobre el valor agregado de la comunidad misma y de sus miembros);

6. Un equilibrio entre la familiaridad y el reto en las actividades (un equilibrio entre las actividades que brindan "un nivel o garantía y comodidad" y las que suponen retos y brindan "elementos de emoción y cambio";

7. Un ritmo para la comunidad (ni demasiado rápido, ni demasiado lento).

Para fortalecer la comunidad de práctica alrededor del CILL y para asegurar su éxito, es crucial reflexionar sobre cómo se pueden cumplir estos requisitos en el contexto del Centro de Idiomas de la FUB y más allá.

\section{Conclusión y recomendaciones}

¿Cómo puede un centro de autoaprendizaje de lenguas contribuir a alentar la autonomía? Me gustaría concluir con algunas recomendaciones basadas en mi propia experiencia en CILL:

- Proporcionar un marco pedagógico: para hacer de un centro de autoaprendizaje de lenguas un lugar para alentar 
la autonomía se necesita un marco pedagógico claro y una concepción compartida de la autonomía, que tenga en cuenta las posibilidades del contexto y de sus participantes.

- Discutir la concepción de la autonomía. Para llegar a este entendimiento, se deben alentar las discusiones con el personal del centro de autoaprendizaje de lenguas, docentes y estudiantes, teniendo en cuenta que el desarrollo de la autonomía es complejo y dinámico, y surge " from the interaction of different individuals together and in relationship with others" dentro del contexto institucional y social (Tatzl, 2016: 47). En esta perspectiva, deberíamos poder revisar nuestra propia concepción de la autonomía para negociar un entendimiento común que sea más apropiado para el contexto dado.

- Escuchar a profesores y estudiantes: involucrarse en el camino hacia la autonomía puede generar dudas, ansiedad y frustración antes de llegar a ser inspirador y gratificante. Pero escuchar a estudiantes y a docentes obliga a que expresen sus sentimientos, sus preguntas y también las respuestas que encontraron en el camino y eso, en una visión humanista de la educación, es el primer paso para buscar y brindar apoyo y andamiaje.
- Dar espacio para la experimentación. Un centro de autoaprendizaje de lenguas debe ser un espacio de intercambio y experimentación en el que todos los actores de la comunidad de aprendizaje, profesores, estudiantes y tutores tengan voz y posibilidad de actuar, innoven y evolucionen personal y profesionalmente.

- Crear una comunidad en el entorno educativo. Fomentar los intercambios y el diálogo, reconocer las aportaciones individuales y colectivas, crear y mantener espacios de diálogo entre los diferentes actores.

- Crear oportunidades para intercambiar experiencias y competencias más allá de la institución. Conocer cómo otros centros de autoaprendizaje de lenguas están organizados en otras instituciones, comparar perspectivas y dialogar con colegas e investigadores en el campo de la autonomía.

- Hacer que su centro sea visible para la gerencia y la administración. Hacer que la función del centro dentro

de la provisión educativa sea más visible y convenza a la administración de los beneficios de invertir en estructuras que involucren a estudiantes, conferenciantes y miembros de la comunidad académica y en el desarrollo de más espacios para la autonomía.

\section{Bibliografía}

Benson, P. (2011). Teaching and researching autonomy in language learning (2nd ed.). Harlow, Essex: Pearson Education.

Berndt, A. \& Deutschmann, R.-U (Eds.) (2014). Sprachlernberatung - Sprachlerncoaching. Frankfurt am Main: Peter Lang.

Böcker, J., Saunders, C., Koch, L. \& Langner, M. (Eds.) (2017). Beratung und Coaching zum Fremdsprachenlernen - Konzepte, Qualitätssicherung, praktische Erfahrungen. Beiträge zu einer Arbeitstagung (Hannover 2015). Gießen: Gießener elektronische Bibliothek. http://geb.uni-giessen.de/ geb/volltexte/2017/12596/

Everhard, C.J. (2015). The assessmentautonomy relationship. In C.J. Everhard \& L. Murphy (Eds.), Assessment and autonomy in language learning (pp. 8-34). Basingstoke, UK: Palgrave Macmillan.

Gardner, D. \& Miller, L. (1999) Establishing self-access: From theory to practice. Cambridge, UK: Cambridge University Press.

Gardner, D., \& Miller, L. (2014). The professional identity of SALL practitioners. In R. Watson-Todd (Ed.), Proceedings of the DRAL2/ ILA Conference (pp. 106-114). Bangkok: King Mongkut University of Technology Thonburi. https://www2 caes.hku.hk/dgardner/files/2015/04/ Gardner Miller 2014.pdf

Holec, H. (1981). Autonomy in foreign language learning. Oxford, UK: Pergamon.

Holec, H. (2009). Autonomy in language learning. A single pedagogical paradigm or two? In F. Kjisik, P. Voller, N. Aoki \& Y. Nakata (Eds.), Mapping the terrain of learner autonomy: Learning environments, learning communities and identities (pp. 21-47). Tampere: Tampere University Press.

Jiménez Raya, M. (2017). Teacher autonomy and agency: The space of possibility in overcoming external obstacles and internal resistances. In M. Jiménez Raya, J.J. Martos Ramos \& M.G. Autor (Eds.), Learner and teacher autonomy in higher education: Perspectives from modern language teaching (pp. 15-35). Frankfurt am Main: Peter Lang.

Jiménez Raya, M., Lamb, T. \& Vieira, F. (2017). Mapping autonomy in language education. A framework for learner and teacher development. Frankfurt am Main: Peter Lang.

Little, D. (1991). Learner autonomy 1: Definitions, issues and problems. Dublin: Authentik.

Little, D. (2001a). We're all in it together: exploring the interdependence of teacher and learner autonomy. In L. Karlsson, F. Kjisik \& J. Nordlund
(Eds.), All together now. Papers from the 7th Nordic Conference and Workshop on Autonomous Language Learning (Helsinki, September 2000) (pp.45-56). Helsinki: University of Helsinki Language Centre.

Little, D. (2001b). How independent can independent language learning really be? In J. Coleman, D. Ferney, D. Head \& R. Rix (Eds.), Language-learning futures: Issues and strategies for modern languages provision in higher education (pp. 30-43). London, UK: CILT in association with the SCHML 2001.

Little, D. (2015). University language centres, self-access learning and learner autonomy. Recherche et pratiques pédagogiques en langues de spécialité, Vol. XXXIV (1). http://apliut.revues. org/5008, 7.03.2016

Littlewood, W. (1996). Autonomy: an anatomy and a framework. System, Vol. 24(4), 427-435.

Littlewood, W. (1999). Defining and developing autonomy in East Asian contexts. Applied Linguistics, Vol. 20(1), 71-94.

Martinez, H. (2008). Lernerautonomie und Sprachenlernverstädnis. Eine qualitative Untersuchung bei zukünftigen Lehrerinnen und Lehrern romanischer Sprachen. Tübingen: Narr.

Mercer, S. (2014). The self from a complexity perspective. In S. Mercer 
\& M. Williams (Eds.), Multiple perspectives on the self in SLA (pp.160176). Bristol, UK: Multilingual Matters.

Oxford, R. L. (2003). Toward a more systematic model of L2 learner autonomy. In D. Palfreyman \& R.C. Smith (Eds.), Learner autonomy across cultures (pp. 75-91). Hampshire, NY.: Palgrave Macmillan.

Paiva, V.L.M. de O., \& Braga, J.C.F (2008). The complex nature of autonomy. Revista D.E.L.T.A., 24 (especial), 441-468.

Stewart, A. \& Irie, K. (2012). Realizing autonomy: Contradictions in practice and context. In K. Irie \& A. Stewart (Eds.), Realizing autonomy. Practice and reflection in language education contexts (pp. 1-17). Basingstoke, UK: Palgrave Mcmillan.
Tassinari, M. G. (2010). Autonomes Fremdsprachenlernen: Komponenten, Kompetenzen, Strategien. Frankfurt am Main: Peter Lang.

Tassinari, M. G. (2016). Ein Selbstlernzentrum als Weg zur Autonomie: Chancen und Herausforderungen. Jahrbuch Deutsch als Fremdsprache. Intercultural German Studies, 40 (2014), 269-293.

Tassinari, M. G. (2017a). A self-access centre for learners and teachers: Promoting autonomy in higher education. In M. Jiménez Raya, J. J. Martos Ramos \& M. G. Autor (Eds.), Learner and teacher autonomy in higher education: Perspectives from modern language teaching (pp. 183208). Frankfurt am Main: Peter Lang.

Tatzl, D. (2016). A systemic view of learner autonomy. In C. Gkonou, D. Tatzl \& S. Mercer (Eds.), New directions in language learning psychology (pp. 39-

53). Switzerland: Springer International Publishing.

Wenger, E. (1998). Communities of practice. Learning, meaning and identity. Cambridge, UK: Cambridge University Press.

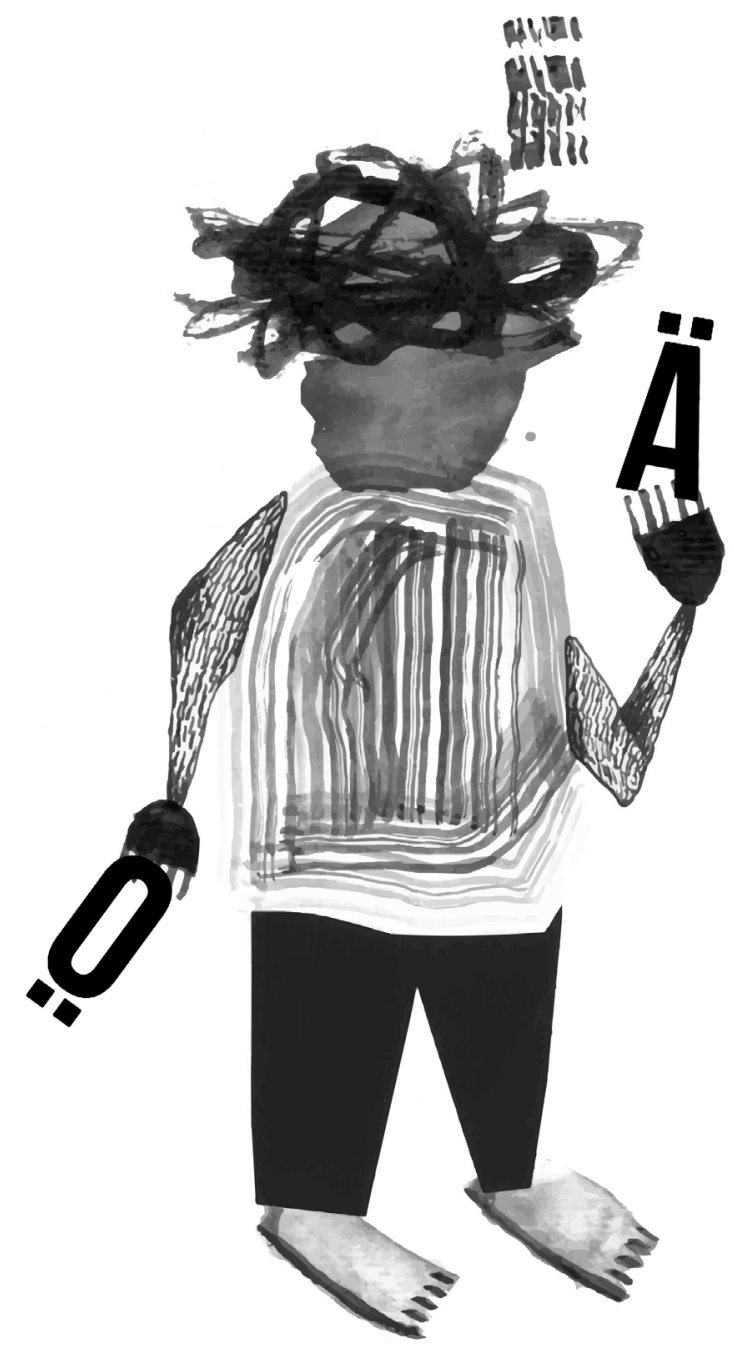

